\title{
Roll-To-Roll Printing of Wearable Sensors and Devices for Personalized Health Monitoring
}

\author{
Mehdi Riza and Xian Du* \\ Department of Mechanical Engineering, University of Massachusetts Amherst, USA
}

*Corresponding author: Xian Du, Department of Mechanical Engineering, Center for Personalized Health Monitoring (CPHM), Institute for Applied Life Sciences (IALS), University of Massachusetts Amherst, USA.

To Cite This Article: Xian Du, Roll-To-Roll Printing of Wearable Sensors and Devices for Personalized Health Monitoring. 2020 - 7(6). AJBSR. MS.ID.001208. DOI: 10.34297/AJBSR.2020.07.001208.

Received: 䨾 February 11, 2020; Published: 觜 March 04, 2020

\begin{abstract}
Personalized Health Monitoring (PHM) is a growing field in the medical community that is intended to address the needs of patients requiring regular monitoring of biodata from a distance. Successful efforts have been made in recent years to solve this problem using wearable electronic sensors/devices. The most effective designs utilize flexible electronics: sensor arrays mounted on polymer substrates that can be wrapped on the skin. While immensely useful due to their inherent advantages in conformability, comfort, and lightweight structures, the challenge lies in finding effective ways to manufacture these devices on a mass scale. Roll-to-Roll (R2R) printing promises high-throughput, high-fidelity fabrication via multiple different methods of imprinting conductive patterns on the substrate. This review examines the applications of these different methods and some of the results that have been demonstrated thus far.
\end{abstract}

Keywords: Additive Manufacturing, Flexible Electronics, Health Monitoring, Medical Devices, Roll-To-Roll Printing, Wearable Sensors, Polydimethylsiloxane, Polymeric Substrates, RFID electronics, Silver Electrodes

Abbreviations: CNT: Carbon Nanotubes; $\mu$ CP: Microcontact Printing; NIL: Nanoimprint Lithography; PDMS: Polydimethylsiloxane; PHM: Personalized Health Monitoring; R2R: Roll-to-roll; UHF RFID: Ultra-high frequency Radio Frequency Identification

\section{Introduction}

Humans have long enjoyed the utility of wearable medical devices since the invention of the first pair of eyeglasses in Northern Italy, around 730 years ago [1]. Only three centuries later, the first hearing aids (ear trumpets) were used to assist the partially deaf in Europe. Founded on simple material properties and ergonomic principles, these devices are examples of passive technological aids that greatly enhanced the quality of life of the wearer while minimizing obstruction into daily activities. In recent years the development of new additive manufacturing methods, especially Roll-to-Roll (R2R) flexible electronics printing technologies, and the miniaturization of computational platforms have unlocked the potential for wearable medical technology that fulfills considerably more advanced functions.

Researchers and engineers around the world have already developed prototypes of different wearable electronic devices that incorporate sensors, microfluidics, and even haptic feedback for personalized patient applications. As with any emergent technology, developing intelligent fabrication methods is essential to reduce the cost of these products and maximize the accessibility to the public. R2R allows large-scale manufacturing of these and other kinds of flexible wearable devices [2-4]. Among the primary applications of wearable electronics is Personalized Health Monitoring (PHM) $[5,6]$. Oftentimes patients with non-critical conditions that require frequent monitoring are unable to receive on-time regular checkups due to factors such as distance from clinics, scheduling conflicts, and general inconvenience (Figure 1) [5].

In such cases, wearable monitoring systems such as the one developed at the Tyndall National Institute in Ireland (Figure 1) can be highly useful for physicians who are able to track their patients' biometric readings every 30 minutes, allowing them to respond in case of an emergency [5]. However, the electronics and substrates are rigid, and unwieldy-this is where flexible electronics can make a large contribution. Sensor electronics, whether optical, chemical, 
physical, or other, are either embedded into or printed on flexible polymeric substrates which are then placed in contact with the skin [6]. Figure 2 shows a capacitive pressure sensor array embedded in a thin elastomeric "skin-like" membrane. In this configuration, the brittleness of the silicon-based sensors is negated by their being imprinted onto the Polydimethylsiloxane (PDMS) film. Researchers note the potential for this device as a form of touch-sensitive artificial skin [7]. Using a similar concept, other groups have developed flexible sensors that detect a wide variety of biomarkers and are capable of real-time data transmission [7-9].
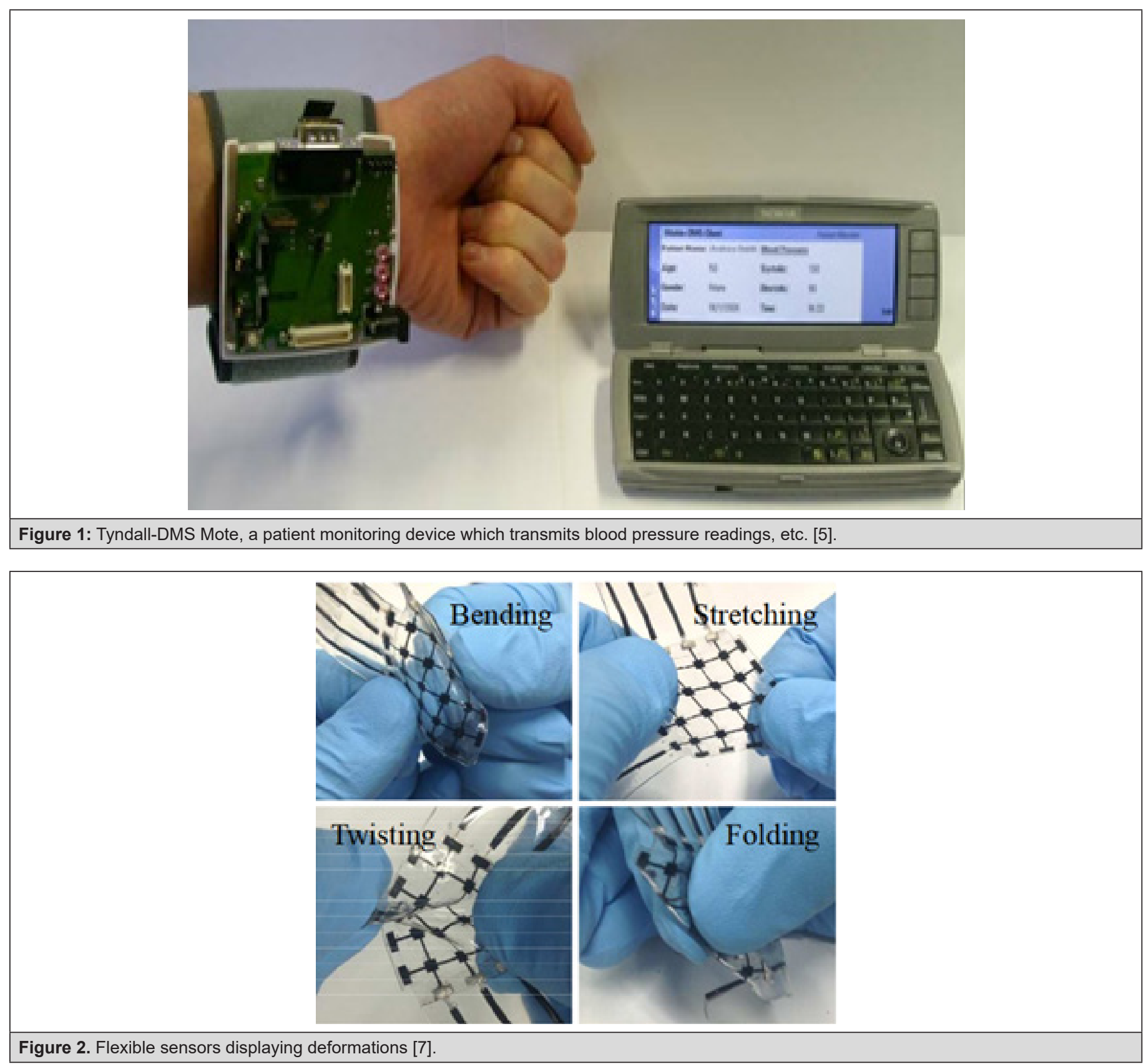

The device in this example was created via microcontact printing, a form of soft lithography in which the Polydimethylsiloxane (PDMS) substrate is stamped with a conductive ink pattern $[10,11]$. The ink is generally made using some form of gold or silver, but research has demonstrated the effectiveness of carbon nanotubes as a high-performance medium too [12]. A major advantage of contact-based lithographic methods such as microcontact printing
( $\mu \mathrm{CP}$ ) or Nanoimprint Lithography (NIL) is that they are scalable for bulk manufacturing [2]. The difference between the two methods is that NIL utilizes thermal imprinting [13]. This can be done by integrating the stamp/press into an R2R system, similar in layout to the old process for printing newspapers $[2,14]$. Figure 3 shows an example of this in an R2R NIL machine for thermal imprinting polymer film [13]. 
Another type of device is mounted directly on the skin like a tattoo, sandwiched between ultra-thin polymer layers [14,15]. Ultra-High Frequency Radio Frequency Identification (UHF RFID) is used in many applications for human monitoring, and one research group used inkjet printing to deposit silver nanoparticle inks onto tattoo paper so they can be easily transferred to the skin. These tattoo tags are ultra-thin $(1-5 \mu \mathrm{m})$ compared to screen or etched tags (tens of $\mu \mathrm{m}$ ) [14]. This technology has a great advantage over current forms of human monitoring devices that utilize bulky wrist/armbands or ankle bracelets containing the RFID electronics.
Skin-like ultra-thin tags may be placed on the body with minimal to no discomfort or interference to the user's normal movement patterns [14].

This technology is not limited to RFID monitoring, as inkjetprinted electrodes used for biosensing of molecules (e.g. cortisol) have already been designed and tested. Inkjet printing has even been used to deposit gold and silver electrodes onto polymeric substrates that had already been imprinted with microfluidic channels by NIL, making composite devices [3].

\section{Print Methods Comparison}

\begin{tabular}{|c|c|c|c|c|}
\hline \multicolumn{5}{|c|}{ Type of Printing } \\
\hline & Screen printing & Inkjet & Lithography ( $\mu \mathrm{CP}, \mathrm{NIL})$ & Flexography \\
\hline Applications & $\begin{array}{l}\text { Voice recognition module on thin } \\
\text { film (polyimide). Large area flexible } \\
\text { sensor array on thin film. Graphene } \\
\text { high res patterned flexible circuit. }\end{array}$ & $\begin{array}{l}\text { Passive UHF RFID skin } \\
\text { tattoo tags. Sensing elec- } \\
\text { trodes for electrochemi- } \\
\text { cal analysis }\end{array}$ & $\begin{array}{l}\text { Microfluidic devices on polymer } \\
\text { substrate, Cellulose biosensing } \\
\text { films. The elastomeric sensor } \\
\text { array on PDMS }\end{array}$ & $\begin{array}{l}\text { CNT array sensors (optical, } \\
\text { chemical tactile) on the ul- } \\
\text { trathin polymer membrane }\end{array}$ \\
\hline Pros & $\begin{array}{l}\text { Low cost; high potential throughput. } \\
\text { Large area; Simplicity }\end{array}$ & $\begin{array}{l}\text { Relatively low cost; } \\
\text { versatile choice of ink/ } \\
\text { substrates }\end{array}$ & $\begin{array}{l}\text { Bulk-scaling potential; very } \\
\text { high speed; high fidelity pattern }\end{array}$ & $\begin{array}{l}\text { CNT stamp microstructures } \\
\text { are resistant to roof collapse }\end{array}$ \\
\hline Cons & $\begin{array}{l}\text { Low-resolution patterns compared } \\
\text { to photolithography and etching. } \\
\text { Limited materials }\end{array}$ & $\begin{array}{l}\text { Slower speed compared } \\
\text { to contact-based meth- } \\
\text { ods; Inefficient ener- } \\
\text { gy-wise }\end{array}$ & $\begin{array}{l}\text { Requires complex feedback } \\
\text { control system to manage print } \\
\text { forces and alignment at the } \\
\text { microscale }\end{array}$ & $\begin{array}{c}\text { Slow speed; limited for } \\
\text { large area stamps. requires } \\
\text { complex fabrication of nano } \\
\text { porous stamps }\end{array}$ \\
\hline Refs & [16-18] & {$[3,4,14]$} & {$[2,3,7,13]$} & [15] \\
\hline
\end{tabular}

Table Abbreviations: UHF RFID: Ultra-High Frequency Radio Frequency Identification; PDMS: Polydimethylsiloxane; CNT: Carbon Nanotube

Table 1 provides a visual aid to summarize the print methods that have been most successful at fabricating flexible electronic devices for PHM applications thus far. The intent is not to convey that one print method is superior in all aspects to others, but to demonstrate that different methods have varying levels of effectiveness depending on the applications. For example, due to inherent limitations screen printing methods will never match the high-resolution of inkjet printing $[4,16]$. However, screen printing is vastly superior for a large area, high throughput fabrication of lower resolution electronics-as well as being much more energyefficient. Meanwhile, lithographic methods such as $\mu \mathrm{CP}$ can produce super-high-resolution patterns in large areas at high speed. But since they are contact-based and rely on premade stamps, they still lack the customizability of inkjet printers which can be easily reprogrammed to produce different patterns [3].

\section{Conclusions and Future Perspectives}

Wearable sensors printed onto polymeric substrates have major advantages over outdated rigid designs due to their low cost, lightweight, flexibility, and comfort [6]. The emergence of higher throughput, lower-cost manufacturing methods for flexible electronics will have a great impact on the field of Personalized Health Monitoring. This paper has gone over the most viable methods used to fabricate these devices in large quantities and explored the capabilities of each. A combination of methods both contact and non-contact (i.e. $\mu \mathrm{CP}$ and inkjet) could be integrated into an R2R system that would be capable of fabricating a wide range of devices on a large scale. This composite system would utilize the best features of each of the components-i.e. the processing speed and high resolution of $\mu \mathrm{CP}$ combined with the adaptability of inkjet. Other print methods such as flexography or screen printing could also be included. A prototype of such a system should be designed and tested with the results being the subject of further study.

\section{Acknowledgment}

Thanks are owed to the researchers and authors of the various groups cited; whose work enabled the writing of this review.

\section{Conflict of Interest}

The author declares no conflict of interest. This work is supported in part by the National Science Foundation (grant no. CMMI1916866 and CMMI1942185). Any opinions, findings and conclusions or recommendations expressed in this material are those of the authors and do not necessarily reflect the views of the National Science Foundation.

\section{References}

1. Ilardi V (2007) Renaissance Vision from Spectacles to Telescopes, American Philosophical Society, Philadelphia, USA. 
2. Kooy N, Mohamed K, Pin LT, Guan OS (2014) A review of roll-to-roll nanoimprint lithography. Nanoscale Res Lett 9(1): 320.

3. Zhou Y (2019) Direct Printing/Patterning of Key Components for Biosensor Devices. Doctoral Dissertation, Univ of Massachusetts Amherst, MA, USA.

4. Abbel R, Teunissen P, Rubingh E, van Lammeren T, Cauchois R, et al (2014) Industrial-scale inkjet-printed electronics manufacturingproduction up-scaling from concept tools to a roll-to-roll pilot line. Transl Mater Res 1(1): 015002.

5. Donoghue J, Herbert J, Stack P (2020) Remote Non-Intrusive Patient Monitoring. Tyndall National Institute, Cork, Ireland.

6. Khan S, Ali S, Bermak A (2019) Recent Developments in Printing Flexible and Wearable Sensing Electronics for Healthcare Applications. Sensors Basel 19(5): 1230.

7. Woo SJ, Kong JH, Kimb DG, Kim JM (2014) A Thin All-Elastomeric Capacitive Pressure Sensor Array based on Micro-Contact Printed Elastic Conductors. Journal of Materials Chemistry C 2(22): 4415-4422.

8. Shiwaku R, Matsui H, Nagamine K, Uematsu M, Mano T, et al. (2018) A Printed Organic Circuit System for Wearable Amperometric Electrochemical Sensors. Sci Rep 8: 6368.

9. Tricoli A, Nasiri N, De S (2017) Wearable and miniaturized sensor technologies for personalized and preventive medicine. Adv Funct Mater 27(15): 1605271

10. Du X, Hardt D, Anthony B (2019) Real Time Imaging of Invisible MicronScale Monolayer Patterns on a Moving Web Using Condensation Figures. IEEE Transactions on Industrial Electronics 67(5): 4077-4087.

11. Chris M, Du X, Hardt D, AlQahtani H (2015) Roll-to-Roll Microcontact Printing of Flexible Aluminum Substrates Using Octadecylphosphonic Acid (ODPA). ASME International Mechanical Engineering Congress and Exposition, Houston, Texas, USA p.7.
12. Volder MFD, Tawfick SH, Baughman RH, Hart AJ (2013) Carbon Nanotubes: Present and Future Commercial Applications. Science 339(6119): 535-539.

13. Mäkelä T, Haatainen T, Ahopelto J, (2011) Roll-to-roll printed gratings in cellulose acetate web using novel nanoimprinting device. J Microelectron Eng 88(8): 2045-2047.

14. Romaguera VS, Ziai MA, Oyeka D, Barbosa S, Wheeleret JSR, et al. (2013) Towards inkjet-printed low-cost passive UHF RFID skin mounted tattoo paper tags based on silver nanoparticle inks. Journal of Materials Chemistry 1(39): 6395-6402.

15. Kim S, Sojoudi H, Zhao H, Mariappan D, McKinley GH, et al. (2016) Ultrathin high-resolution flexographic printing using nanoporous stamps. Science Advances 2(12): 1601660.

16. Chang WY, Fang TH, Lin HJ, Shen YT, Lin YC (2009) A Large Area Flexible Array Sensors Using Screen Printing Technology. J Display Technol 5(6): 178-183.

17. Numakura D (2008) Advanced Screen-Printing Practical Approaches for Printable \& Flexible Electronics. $3^{\text {rd }}$ International Microsystems, Packaging, Assembly \& Circuits Technology Conference, Taipei, Taiwan.

18. Hyun WJ, Secor EB, Hersam MC, Frisbie CD, Francis LF (2015) HighResolution Patterning of Graphene by Screen Printing with a Silicon Stencil for Highly Flexible Printed Electronics. Advanced Materials 27(1): 109-115. 